\title{
Wireless Sensor Network for Localized Maritime Monitoring
}

\author{
P. Barbosa N. M. White N. R. Harris \\ Electronic Systems and Devices Group \\ School of Electronics \& Computer Science \\ University of Southampton, UK
}

\begin{abstract}
In this paper, we propose a novel routing algorithm for maritime monitoring of short term events where large area monitoring relies on small buoys drifting along with local currents. The objective of this algorithm is to improve communication where scalable mobile networks are optimized by taking into consideration a weight function based on remaining energy and relative distance between buoys. To improve wide area communication, amplified transmitters were selected. Therefore route optimization requires a different approach, as transmission requires more power than reception. We present two new approaches for message routing, where relay selection depends on a weight advertised periodically, and calculated through remaining energy and route distance to sink. The first approach uses a single route, while the second approach balances its load by using different routes selected in proportion to route weight. In both cases, the algorithms show improvements over singlehop and greedy approaches. Moreover, the second solution shows a more efficient message distribution through relay nodes that ultimately results in lower latency.
\end{abstract}

\section{Introduction}

Traditionally, marine monitoring is performed by satellites and airborne sensors. Satellites have fixed trajectories and can provide monitoring over a wide region, while airborne surveillance is a temporary but more flexible method. Both satellites and airborne methods are able to measure position, extent and shape by using Synthetic Aperture Radar colour analysis. Other information can be derived from it, such as local wind and wave data.

We propose a novel approach to maritime monitoring that uses floating Wireless Sensor Networks (WSNs) to constantly monitor a measurand. The WSN is built by small nodes randomly deployed on water surface which drift with the current to follow the event. Wireless Sensor Networks (WSNs) are solutions where cables are replaced by wireless communication fitted into small intelligent devices called nodes [2]. WSNs can operate unattended in any environment and location, including those where human presence is risky or even impossible. The data collected can then be sent to users or devices located remotely in a periodic or on-demand manner.

Oil slicks present different challenges to WSNs: they can be extremely dynamic, with variable size and can happen in anywhere around the world. In addition, a local monitoring mechanism could allow better real-time understanding of cleaning procedures performance. Given the potential size of the region to be monitored, nodes fitted with amplified transceivers for extended communication range are seen as potential solution. Yet, to balance the extra energy required by the transceiver, ultra low power processors with limited processing abilities are preferred. As such, two new approaches to routing algorithms were conceived to optimize a weight function based on relative distance between nodes and remaining energy.

\section{$1.1 \quad$ Oil slick monitoring}

Petroleum products are transported all around the world everyday, mainly by pipelines and oil tankers. Occasionally, accidents occur and oil is spilled, potentially causing serious impact to ecosystems and economies. This is particularly serious in maritime spills, as they can damage both coastline and the sea bed, affecting the ecosystem for several years.Knowing the slick location, movement and characteristics, such as thickness or chemical composition, may help achieve a more effective response to the problem. Moreover, when applying cleaners such as dispersants, it is helpful to have constant monitoring on slick thickness, as to avoid excess spread that can increase the danger of contamination due to the dispersants.

So far, oil spill tracking is being performed mainly through satellite image processing or airborne sensors [10]. Among sensors used the most common are infrared/ultraviolet, laser and Synthetic Aperture Radar (SAR) [8]. However, each technique has its flaws: some are un- 
able to monitor thickness (ultraviolet, for example), while others need wind within a certain range (such as SAR). Potential sources of interference include atmospheric absorption, clouds, surface roughness, wind speed, algae and other suspended matter, glacial flour and biogenic oils.

\subsection{Related work}

WSNs have already been deployed on the ocean. The SECOAS project [4] was aimed at utilizing a WSN with fixed nodes for sea bed monitoring near a wind farm. Nittel et al [9] explore communication connectivity and sensing uniformity effects from sensor deployment strategies. Garcia et al. [6] studied positioning for underwater acoustic networks. In all cases, communication is one of the most important aspect when designing WSNs for maritime monitoring. Location can be remote, therefore the usability of the system will depend on its ability to communicate and supply the requested data. Argos transmitters [11] provide remote sensing using satellite communication. Currently they support GPS location, sea surface temperature, location, and other measurements. Genesis alert system [5], on the other hand, is a different approach to monitoring. It consists of stationary sensing buoys with wireless communication that were designed to be deployed in small numbers.

\section{Wireless sensor Networks}

Monitoring hazardous spills on the sea, namely oil slicks, can be seen as a complete example of WSN deployment: disasters can occur anywhere, at any time, possibly in harsh environments (somewhere on the ocean and with severe weather), the spilled material will drift according to winds and streams and its shape and size will change continuously, possibly stretching and dividing. Hence, maritime monitoring has impact on all the design areas of WSNs: sensing, processing, communication and energy. Currently, to the best of our knowledge, there is no implementation of WSN for oil slick measurement, and there are two potential parameters to be sensed: oil slick thickness and composition. The information about these parameters give good indications to assess which clean up methods to apply. Given the flexibility of these networks, different information can be retrieved by adding new sensors, such as temperature, salinity or wind speed, making this solution effective for a broad type of maritime monitoring applications.

In WSNs, message delivery and optimized energy consumption are two of the most important aspects. The resource-constrained networks and the distributed nature of these networks make conventional protocols impractical. Nodes must be able to operate for extended periods of time, and given the small size of the devices, energy available is a major restriction. On the other hand, the application-aware nature of WSN means that performance shouldn't be measured in data rate, but in network lifetime to perform the required tasks in the most reliable manner [13]. Routing protocols and data aggregation mechanisms have also been developed to optimize energy savings and communication by transmitting messages to specific destinations. In addition, one of the main concerns when designing the network has to do with environmental pollution. Usually nodes rely on batteries to provide energy, which can be toxic to the marine environment. To avoid the use of these energy sources, ultracapacitors are a potential solution, as they do not use any toxic components. The main differences between ultracapacitors and batteries are that ultracapacitors have a lower energy storage capacity and higher leakage current than batteries. Due to this, it is important to consider energy harvesting mechanisms. These generate electric energy from light, thermal or kinetic energy sources which can be used to recharge the ultracapacitor and increase node lifetime or even allow continuous operation. By utilizing renewable power supplies, the routing algorithm must be energy-aware. Also, and to balance differences between nodes, it must analyse and take advantage of energy across the network. This is particularly true given the extended range required to achieve better coverage of the sensed region.

\subsection{Routing algorithms for Wireless Sen- sor Networks}

One of the most common assumptions that leads to new routing algorithms and protocols is that by avoiding long range, singlehop communication, it is possible to save energy from the transmitter, even if transmitter and receiver are within range of each other. Therefore algorithms try to search for ways to relay messages through other nodes between the origin and destination in a multihop manner. It is known that transmission power increases with the square of distance, therefore relaying one message through at least one node inbetween origin and destination should in principle enable energy saving, although in practice it is not as straightforward as this.

Clustering is one of the routing techniques for WSN. It consists of dividing the whole network into smaller regions, called clusters. In each cluster a node is defined to become the leader, also called Cluster Head $(\mathrm{CH})$ or broker. Depending on the network, the $\mathrm{CH}$ can be a regular node that becomes leader after being elected, or a resource-rich node that is placed specifically for that purpose. When a $\mathrm{CH}$ is defined, it takes responsibility of organizing and managing sensing and communication inside the cluster, receive data from nodes and forward it to any destination outside the cluster. Clustering solutions present advantages over other solutions [1]: they are scalable, can manage bandwidth very 
efficiently, reduce overhead for topology maintenance and allow energy savings by means of TDMA/FDMA methods and data aggregation.

\section{Dynamic algorithm for maritime monitor- ing}

Oil slick size can vary dramatically both in shape and size. This makes static algorithms unsuitable. The best alternative to improve communication would be to send messages to $\mathrm{CH}$ without intermediates. However, as this distance can extend up to several miles, it is preferable to route them through relays to keep the energy usage low. Yet, it is not feasible to have multiple relays, as it increases the number of buffered messages across the network. In addition, the potential number of nodes possible to achieve in a large slick can extend up to thousands, where to make the concept plausible low-cost, low-power devices need to be used. Therefore, the routing algorithm must be simple enough to be implemented in low power hardware. At the same time it must be flexible to adapt to different network sizes and dimensions. To that extent, clustering protocols appear as the best alternative, as they can be highly scalable and allow localized coordination. We start by defining intracluster communication, where one special node controls all communication in its region.

Given the node distribution is dynamic (they move according to current and wind), the network topology will change unpredictably. The routing algorithm must adapt throughout time to physical constrains so inter-node communication is not severely affected. The dynamic protocol described below is therefore a feasible approach to the challenges present as it is constrained to a two-hop message relay, which allows energy savings among nodes. A twohop delay was chosen since it provides improved coverage, while minimizing message delay. In singlehop communication, delay is minimal, as it is only depending on channel availability. In contrast, multihop communication delay increases linearly with the number of hops for the lightest load [12]. One message can be relayed through several nodes or, in the worst case scenario, through every node. In a network with $N$ nodes arranged sequentially where each node is able to transmit once every period $T$, a message generated by node $N$ will need $N$. T time to travel to the $\mathrm{CH}$.

\subsection{Routing algorithm}

The starting point consists of a region where $N$ nodes are randomly deployed around the $\mathrm{CH}$, a resource-rich node that manages how the nodes inside the cluster perform their sensing and communication tasks. All nodes are within range of the $\mathrm{CH}$. The region is divided into two areas, as shown in figure 1: the area inside $R_{A}$ radius, which is closer to $\mathrm{CH}$, and the area outside $R_{A}$. If nodes are closer to the centre, within $R_{A}$ radius, they may advertise themselves in order to act as a relay node. If nodes are outside $R_{A}$, they can select whether to communicate directly or to use another node as a relay, upon receiving advertising from closer nodes.

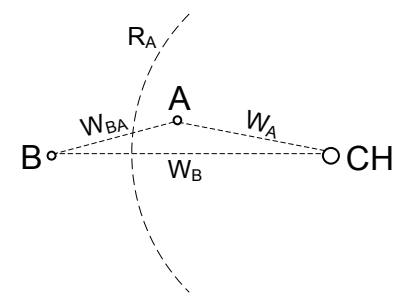

Figure 1. Displacement of nodes around $\mathrm{CH}$

Advertising events are performed periodically upon $\mathrm{CH}$ command. After receiving an advertisement from the $\mathrm{CH}$, each node compares its current $\mathrm{CH}$ to the advertised one. If it is different and the newly received $\mathrm{CH}$ is a better alternative, the node changes its routing table so it directs its message to it. Once the node's $\mathrm{CH}$ has been defined, it decides whether it will advertise itself, based on $R_{A}$. If the distance is smaller than $R_{A}$, it advertises itself and waits for messages from the vicinity. If the distance is larger than $R_{A}$, then it simply waits for advertisements from other nodes. $R_{A}$ is a system designed parameter, established to be half the maximum communication range, as this is the equal power usage point to send a message using two-hop relay.

The advertisement message comes with route weight information. This weight is calculated by the advertising node, based on communication distance and energy left in battery:

$$
W_{X Y}=\left(1-\frac{E_{r e m}}{E_{\max }}\right) \operatorname{Dist}_{X Y}^{2}
$$

where $W_{X Y}$ is the advertised weight, $E_{r e m}$ is the remaining energy in node $X, E_{\max }$ is the maximum potential value of energy in node $\mathrm{X}$ and $\operatorname{Dist}_{X Y}$ is the estimated distance between the $\mathrm{CH}$ and the advertising node. This equation was derived empirically and does not represent any optimization. Distance is raised to the square since power increases approximately to the square with distance. Linear battery discharge is a good approximation for lithium batteries. 1, upon receiving weight values, node B updates its communication weight and calculates the weight of transmitting through $\mathrm{A}$, being $W_{B}$ the direct weight and $W_{B A}+W_{A}$ the weight of using A as relay. 


\section{$\mathrm{O}$ \\ Figure 2. Example of weights in different paths}

\subsection{Single relay decision}

Single Relay Decision (SRD) uses the knowledge of total route weights to decide the best alternative. After advertisements from other nodes, a node receiving these messages compares them and selects the best route, calculating the best weight function as the minimum between current and advertised weights. The route selected will be valid until one of the two following conditions occurs: (1) $\mathrm{CH}$ sends an advertisement beacon or (2) the relay node energy source becomes depleted and advertises its condition. When $\mathrm{CH}$ sends a beacon, the node becomes obliged to send messages directly to $\mathrm{CH}$ until a better route is advertised. The same thing happens if the relay node becomes depleted. In that case, the relay node saves the little remaining energy to advertise its shutting down and route whichever messages are still present in its queue. Figure 2 shows an example where node $\mathrm{O}$ receives route advertisements from $\mathrm{A}, \mathrm{B}$ and $\mathrm{C}$. It then calculates the weight to each node and adds it to the advertised weight. The route path selected is the one that presents the lowest weight.

\subsection{Multiple relay decision}

Multiple Relay Decision (MRD) protocol provides a more complete alternative to SRD. This algorithm compensates the greediness of single relay by distributing the transmission more evenly through routes that present better weights than singlehop to $\mathrm{CH}$. It uses the total weight to distribute messages more evenly through different routes. This distribution resembles the El Farol Bar Problem [3], a game theory problem where the inhabitants of Santa Fe, New Mexico decide whether they should go to El Farol Bar on Thursday night. In our approach, we implement a simple inductive reasoning method based on path weight. If a node has low weight path, it is more likely that many nodes will select it as a route, whereas another node with weightier path (even if for a small difference) won't be selected at all until new advertisement. Knowing route weights for a message to reach the $\mathrm{CH}$, a local decision should be made in order to balance the load across different nodes.

To balance message load, each node chooses randomly between the relays in its routing table which node will route each message, based on a proportionality of their weights, i.e., if route $X$ 's weight is half the weight of route $Y$, the probability of being selected is twice as high. Another inherent aspect of this approach is that the message distribution will reduce latency, as the probability of each node getting its message queue full is smaller. Figure 2 shows an example node distribution where node $\mathrm{O}$ can consider relaying messages to the $\mathrm{CH}$ through three advertised nodes or direct communication. There are three possible paths adding to the direct one, being $W_{A}=a_{1}+a_{2}, W_{B}=b_{1}+b_{2}$, $W_{C}=c_{1}+c_{2}$ and $W_{D i r}=d$. As an example, if $W_{A}=2 . W_{B}$, then the probability of path $\mathrm{B}$ being selected is half the one of path $\mathrm{A}$.

Each node outside $R_{A}$ resets its routes when receiving advertisements from the $\mathrm{CH}$ and waits for nodes within $R_{A}$ to advertise their route weight calculated using equation 1 . After receiving the advertisements, nodes will select the best three relayed routes and add them to the direct one.

\section{Simulation}

By balancing more traffic to lighter routes, nodes are expected to achieve best overall energy distribution. To demonstrate this, a simulator was developed where 100 stationary nodes are randomly deployed around the $\mathrm{CH}$. Assumptions were made to execute the simulation: $\mathrm{CH}$ is a resource-rich node; all nodes are deployed in a circular area around $\mathrm{CH}$ and are within communication range of $\mathrm{CH}$; all nodes start with the same energy and that the maximum radius corresponds to maximum transmission power, being $P_{T x}=6 . P_{R x}$. For simplicity reasons, message collisions, interference, message loss, and delays are ignored for the time being. The constraint of no aggregation is the worst case scenario, In this case, we assume each node generates a message every 10 time steps, leaving the other nine steps for message relay.

In order to compare the results achieved, singlehop (SH) and greedy (based on GPSR [7]) protocols were implemented. Singlehop was chosen since it is the best alternative when $P_{T x} \approx P_{R x}$. On the other hand greedy was chosen as it uses the shortest path towards the $\mathrm{CH}$, minimizing energy consumption. The same node distribution was kept for the four different routing algorithms. Parameters and values were as displayed in table 1 .

Each node works as an independent entity and communication between nodes is performed through messages transmitted between them. Being different in concept and requirements, $\mathrm{CH}$ and nodes have different types of messages from each others, as described in table 2 . To improve resources management, nodes only send messages upon request from the $\mathrm{CH}$. Therefore, the $\mathrm{CH}$ has two types of messages: ADV_CH and CH_QUERY. ADV_CH is an advertisement message with $\mathrm{CH}$ identification that allows a node 
Table 1. Simulation parameters

\begin{tabular}{cc} 
PARAMETER & VALUE \\
\hline Number of nodes & 100 \\
Initial energy & $200 \mathrm{~J}$ \\
Maximum $T_{x}$ Energy & $48 \mathrm{~mJ}$ \\
$R_{x}$ Energy & $8 \mathrm{~mJ}$ \\
Max. distance to $\mathrm{CH}$ & approx. 1 mile \\
Max. communication range for nodes & 0.65 miles \\
Advertisement range $\left(R_{A}\right)$ & 0.5 miles \\
Node transmission period & 1 minute \\
New message generation period & Every 10 minutes \\
\hline
\end{tabular}

to decide whether to use this $\mathrm{CH}$ as a sink or maintain the current one. Also, if a node receives an advertisement from the current $\mathrm{CH}$, and as long as its distance to $\mathrm{CH}$ is smaller than $R_{A}$, it can advertise itself to other nodes. CH_QUERY message requests that all assigned nodes, when receiving it, reply back to $\mathrm{CH}$ with sensed values.

Table 2. Nodes messages

\begin{tabular}{cll} 
NODE & MSG. TYPE & DESCRIPTION \\
\hline CH & ADV_CH & $\begin{array}{l}\text { CH advertisement message } \\
\text { CH's request for sensed data from nodes }\end{array}$ \\
& CH_QUERY & Node advertisement \\
Node & ADV_NODE & Tode answer to CH request for sensed data \\
& ADV_SHUTDOWN & $\begin{array}{l}\text { Broadcast message sent by any node just af- } \\
\text { ter reaching the minimum energy level }\end{array}$ \\
\hline
\end{tabular}

Nodes, upon receiving a ADV_CH message, will verify if they are within $R_{A}$ range. If so, they will send a NODE_ADV message to all nodes within communication range. Any node outside $R_{A}$ can then decide which path to use to communicate with $\mathrm{CH}$. To reply to any $\mathrm{CH}$ request each node sends a TO_CH message to its relay node or directly to $\mathrm{CH}$, depending if it is using any relay or not. ADV_SHUTDOWN is used in greedy, SRD and MRD algorithms by nodes that are relaying messages to inform all other nodes that it is nearly depleted and all the other using it must send messages to $\mathrm{CH}$ directly from that moment onwards.

\section{Results and discussion}

As all four algorithms use different methods to communicate with $\mathrm{CH}$, network topologies evolve very differently from each other. It also becomes clear that each algorithm has its particularities, which is apparent from the way nodes become depleted and stop transmitting. To better evaluate this, two comparative methods were used: number of nodes

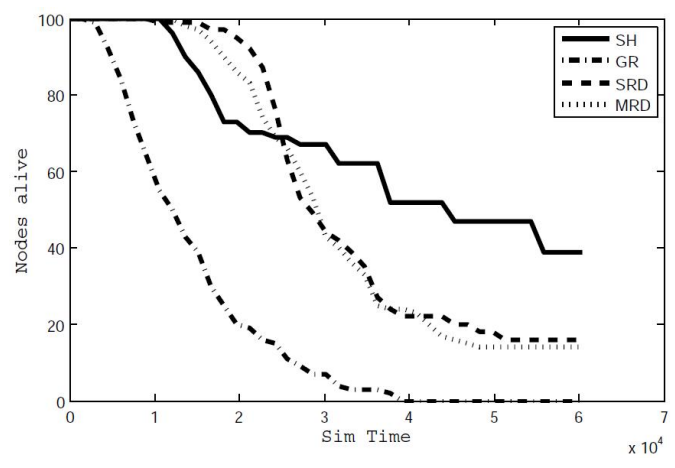

Figure 3. Number of nodes alive

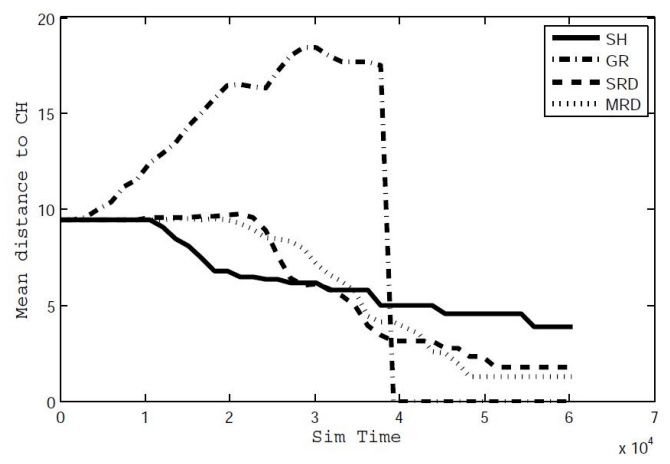

Figure 4. Distance of nodes to $\mathrm{CH}$

alive and mean node distance to $\mathrm{CH}$. The first one shows the rate at which nodes die, giving a good indication of how energy is being used across the network. The second shows what variation there is between dead nodes closer and more distant to $\mathrm{CH}$.

Figure 3 shows the mean nodes alive with each algorithm through time. The increase in lifetime given by SRD and MRD is noticeable when compared to singlehop and greedy algorithms. Also, greedy algorithm never achieves any improvement over any other algorithm. As such, the network keeps a more complete coverage for a longer period using either of the two proposed algorithms.

Figure 4 shows the mean distance between nodes and $\mathrm{CH}$ throughout time. Singlehop nodes start dying from the outside because they require more energy to transmit to $\mathrm{CH}$. The greedy protocol shows a constant increase in mean radius, since nodes closer to $\mathrm{CH}$ are requested to send more messages than nodes located further away, leaving distant nodes with more energy. It is noticeable that both SRD and MRD achieve a more uniform distribution than singlehop and greedy algorithms. MRD also outperforms SRD when nodes start to become depleted, showing that it tries to maintain the energy usage uniform for longer.

Another important aspect of protocols has to do with la- 


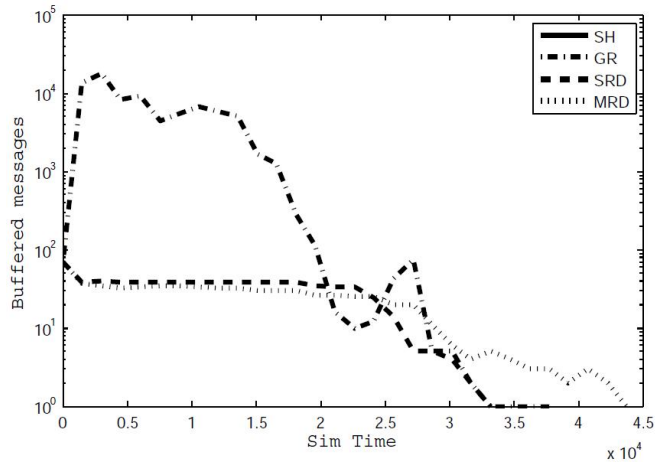

Figure 5. Sum of messages buffered across the network

tency, and this can be estimated by the number of messages queued in each node. Figure 5 shows the sum of buffered messages in all nodes across the network. Singlehop, as expected, has no queued messages. Greedy accumulates messages especially in central nodes and would require a very large buffer in those nodes to avoid any information loss. Although it happens in both algorithms, SRD accumulates more messages in one node when this shows to be best alternative, independently of energy remaining. When the best located node becomes depleted, there is a sudden decrease in the total number of buffered messages. This means that some information may have been lost, since there is not sufficient energy to transmit it. However, to buffer all messages, a large cache would be needed, and if it is not possible to know which node was to be selected as relay (node displacement is random), all nodes would require large buffers. In comparison, MRD won't require such a large buffer, since it has been shown to have better message load distribution.

\section{Conclusions}

WSNs for oil slick monitoring requires a different approach to what's been done so far: lifetime is measured in days or weeks instead of months or years, network size is unknown, high power transceivers are preferred to lowpower and ultra low-power ones and energy source alternatives may have to exclude batteries. As such, a new approach was needed that could improve node coverage and message delivery in a short period of time by efficiently managing relative location and overall energy while keeping the architecture simple and predictable.

Our algorithms show better performance than singlehop and greedy algorithms in simulation. They present improved energy usage and distribution, low message latency and no convergence time is needed for the network to oper- ate. Between the two proposals MRD performs better than SRD. Even if the gain in nodes alive is not as good as SRD, the more uniform energy distribution and lower latency are important aspects. Another important aspect of these solutions is simplicity. Although they do not represent the optimal solution, they can easily be implemented even in resource constrained nodes.

Future work will take into account three different aspects to assess if the algorithms can comply with aspects commonly found in oil slicks: node mobility, energy harvesting and network scalability.

\section{References}

[1] A. A. Abbasi and M. Younis. A survey on clustering algorithms for wireless sensor networks. Comput. Commun., 30(14-15):2826-2841, 2007.

[2] I. Akyildiz, W. Su, Y. Sankarasubramaniam, and E. Cayirci. Wireless sensor networks: a survey. Computer Networks, 38:393-422, 2002.

[3] W. B. Arthur. Inductive reasoning and bounded rationality. In American Economic Review (Papers and Proceedings), number 84, pages 406-411, 1994.

[4] M. Britton and L. Sacks. The SECOAS Project: Development of a Self-Organising, Wireless Sensor Network for Envirnomental Monitoring. In Second International Workshop on Sensor and Actor Network Protocols and Applications (SANPA 2004), August 2004.

[5] EnviroWatch [Online]. Genesis alert system. http://www.genalert.com/, 20 Nov 2007.

[6] J.-E. Garcia, K. Kyamakya, and K. Jobmann. Ad-hoc positioning for ocean sensor networks. Instrumentation and Measurement Technology Conference, 2004. IMTC 04. Proceedings of the 21 st IEEE, 2:1317-1321, 18-20 May 2004.

[7] B. Karp and H. T. Kung. GPSR: greedy perimeter stateless routing for wireless networks. In Mobile Computing and Networking, pages 243-254, 2000.

[8] I. Maciejewska. Real time oil spill detection and tracking based on air-born and satellite remote sensing technologies. Technical report, SPREEX - SPill REsponse EXperience, www.spreex.net [online], last accessed 20 Oct 2007, 2007.

[9] S. Nittel, N. Trigoni, K. Ferentinos, F. Neville, A. Nural, and N. Pettigrew. A drift-tolerant model for data management in ocean sensor networks. In MobiDE '07: Proceedings of the 6th ACM international workshop on Data engineering for wireless and mobile access, pages 49-58, New York, NY, USA, 2007. ACM.

[10] N. Robbe and T. Hengstermann. Latest trends in airborne pollution surveillance. Sea Technology, pages 41-44, October 2007.

[11] Seimac [Online]. RF-700C2 - Combo Oil Spill Tracker. http://www.seimac.com/, 20 Nov 2007.

[12] W. Ye and J. Heidemann. Medium access control in wireless sensor networks. Wireless sensor networks, pages 73-91, 2004.

[13] Q. Zhao, L. Tong, and Y. Chen. Energy-aware data-centric mac for application-specific sensor networks. In Proc. of IEEE Workshop on Statistical Signal Processing, 2005. 